\title{
Effectiveness of miltefosine-pentoxifylline compared to miltefosine in the treatment of cutaneous leishmaniasis in C57BI/6 mice
}

\author{
Ana Angélica Amorim Santarem ${ }^{[1]}$, Gabriel Frizon Greggianin ${ }^{[1]}$, \\ Rafaela Garcia Debastiani ${ }^{[1]}$, Jefferson Bruno Pereira Ribeiro ${ }^{[2]}$, Demerson Andre Polli ${ }^{[3]}$ \\ and Raimunda Nonata Ribeiro Sampaio ${ }^{[1],[2]}$
}

[1]. Laboratório de Dermatomicologia, Faculdade de Medicina, Universidade de Brasília, Brasília, DF. [2]. Pós-Graduação de Ciências Médicas, Faculdade de Medicina, Universidade de Brasília, Brasília, DF. [3]. Departamento de Estatística, Universidade de Brasília, Brasília, DF.

\begin{abstract}
Introduction: The treatment of leishmaniasis ischallenging, given the difficulties in drug administration and resistance. Therefore, we chose to test the efficacy of miltefosine combined with pentoxifylline. Methods: Twenty-seven isogenic C57Bl/6 mice were infected with Leishmania (Leishmania) amazonensis, and equally divided into three groups: miltefosine (200mg/ $\mathrm{kg} /$ day), miltefosine $(200 \mathrm{mg} / \mathrm{kg} /$ day $)$ with pentoxifylline $(8 \mathrm{mg} / \mathrm{kg} /$ day $)$, and untreated. Response to treatment was evaluated using paw diameter and parasitological criteria. Results: The number of viable Leishmania reduced significantly within the miltefosine-pentoxifylline group $(\mathrm{p}<0.05)$. Conclusions: There is hope that a viable treatment exists for Leishmania infection.
\end{abstract}

Keywords: Cutaneous leishmaniasis. Pentoxifylline. Miltefosine.

Cutaneous leishmaniasis (CL) is an infectious disease caused by different species of protozoa from the genus Leishmania, and is observed worldwide. The pathogenesis of CL involves a dysregulated immune response with overproduction of proinflammatory cytokines, regulatory cytokines deficiency, and lysis mechanisms by which intracellular parasites escape ${ }^{1,2}$.

Unfortunately, therapies for treating Leishmania infection remain limited, and pentavalent antimonials have remained the drugs of choice for over 60 years. All first and second drug choices, including miltefosine, have high toxicity, require parenteral use, have high rates of relapse and drug resistance, and ultimately lead to treatment failure, especially in immunocompromised patients who desperately require new therapies ${ }^{3}$. Miltefosine (hexadecylphosphocholine) is an alkylphosphocholine, originally developed for the treatment of cancer, which was discovered to possess antifungal, antiamoebic, and leishmanicidal activity ${ }^{4,5}$. The drug exerts its cytotoxic effect by interfering with the metabolism of membrane phospholipids, altering the composition, permeability, stability, and fluidity of the cell membrane, subsequently inducing apoptosis ${ }^{3,6}$. Miltefosine's mechanism of action also indirectly

\footnotetext{
Address to: $\mathrm{Dr}^{\mathrm{a}}$ Raimunda Nonata Ribeiro Sampaio. SHIS QI 25, Conjunto $2 /$ Casa 01, 71660-220 Brasília, DF, Brasil.

Phone: 5561 3367-1331; Mobile: 5561 8121-6100; Fax: 5561 3367-3825

e-mail: raimunda.sampaio@gmail.com

Received 2 October 2013

Accepted 9 April 2014
}

induces immunomodulatory antileishmanial mechanisms by increasing production of gamma interferon (IFN- $\gamma$ ), tumor necrosis factor alpha (TNF- $\alpha$ ), and interleukin 12 (IL-12), thereby increasing phagocytosis and inducing an immune response biased towards a Th1 profile ${ }^{7,8}$.

Similarly, pentoxifylline (a methylxanthine) has been investigated in the treatment of $\mathrm{CL}$, based on its inhibition of nuclear factor kappa beta (NF-k $\beta$ ), TNF- $\alpha$, and intercellular adhesion molecule 1 (ICAM-1) $)^{8,9}$, as well as its nephroprotective effect when combined with antimonials ${ }^{10}$. These immunomodulatory effects may help to regulating patients' exacerbated and inefficient intracellular immune response to parasites, and subsequently reduce tissue inflammation. Therefore, this study aims to examine the combined immunomodulatory effects of two oral drugs, by comparing miltefosine-pentoxifylline to miltefosine alone.

In this study, we used 27 isogenic C57Bl/6 mice and promastigotes of Leishmania (Leishmania) amazonensis (MHOM/BR/PH8) from the Dermatomycology Laboratory at the University of Brasilia. All mice were born and raised in the vivarium of the Faculty of Medicine at the University of Brasilia. This species was chosen since it is a model parasite that grows easily in culture media, improving study speed and viability. While it is not the most prevalent species, Leishmania amazonensis is distributed throughout the country, where it causes severe clinical manifestations and diffuse cutaneous leishmaniasis. No satisfactory treatment has been discovered ${ }^{11}$.

These experiments were evaluated and approved by the ethics committee for animal use in the Institute of Biological Sciences of the University (UnBDOC 43044/2010). All animals 
were inoculated with $10^{7}$ parasites in metacyclic promastigote form, which were injected subcutaneously in the dorsal tissue of the right footpad, following the standardized technique ${ }^{4}$. Thirty days after inoculation, the mice were divided according to sex (6 females and 21 males) and randomly sorted into 3 groups, each group containing 2 females and 7 males. The groups were labeled and treated as follows: (M) oral miltefosine $(200 \mathrm{mg} /$ $\mathrm{kg} /$ day $),(\mathrm{M}+\mathrm{P})$ oral miltefosine $(200 \mathrm{mg} / \mathrm{kg} /$ day $)$ with oral pentoxifylline $(8 \mathrm{mg} / \mathrm{kg} /$ day), and $(\mathrm{C})$ no treatment. Oral dosage was performed daily for a period of 12 days, using a gavage needle. Treatment response was evaluated using the lateral diameter of the right paw, lymph culture in Novy-MacNealNicolle (NNN) media, the methylthiazol tetrazolium (MTT) dye colorimetric assay, and visual detection of amastigotes.

We measured the lateral-lateral diameter of the right footpad of each mouse, using a Mitutoyo ${ }^{\circledR}$ millimeter caliper, on the first (D1), sixth (D6) and twelfth (D12) day of treatment. On the thirteenth day (D13), lymph was aspirated from the infected paws and cultured in NNN media. Cultures were observed daily, under an inverted light microscope at $40 \times$ magnification, to detect the presence of parasites. The MTT test was carried out using a limiting dilution assay as previously described ${ }^{12}$. In short, the infected paws of 2 male mice from each group were subjected to sterile dissection and trituration in $5 \mathrm{ml}$ of Schneider's culture medium, enriched with $20 \%$ fetal bovine serum. Serial dilution was performed in standard 96 well plates. Plates were incubated at $26^{\circ} \mathrm{C}$ for 4 days, with daily observation under an inverted light microscope. At the end of that period, plates were subjected to the MTT colorimetric assay, as previously described ${ }^{13}$. Each culture assay was performed at the end of treatment (D13) and on D63. On D13, group (C) consisted of 7 mice, group (M) 7 mice, and group (M + P 8) mice. On D63, group (C) consisted of 5 mice, group (M) 3 mice, and group $(\mathrm{M}+\mathrm{P} 4)$ mice.

Statistical analysis: Statistical analysis was performed using R 3.0.0 software (R Core Team, 2013).

Ethical considerations: From the original 27 mice, 9 died prior to the end of the experiment, mainly due to infighting and resulting injuries. Six mice per group were considered adequate for statistical analysis, and the possibility of animal death had been considered at the time of project approval by the Ethics Committee (UnB-Doc 43044/2010).

Paw diameter: Since pad diameter was not normally distributed (based on the Shapiro Wilk and KolmogorovSmirnov tests), we used the Kruskal-Wallis test (using a chisquare distribution) to analyze the three groups' response to treatment. Our analysis found that on D1, the treatment effect was not significant $(p=0.06)$. However, the treatment effect on D6 and D12 was significant $(p<0.01, p<0.01)$. Since the sample size was small, we tested the suitability of the KruskalWallis test using Monte Carlo tests. Unfortunately, we were unable to process a sample calculation for each variable in the analysis. Although the $p$-values were different between the two methods, the inferences were always consistent, so we concluded that use of the asymptotic method did not cause bias in the inference.
Since the Kruskal-Wallis test found no difference between (M) and $(\mathrm{M}+\mathrm{P})$ on D6 and D12, we investigated the differences in pairs, using the Wilcoxon test to analyze the groups head-tohead. Treatment with both $(\mathrm{M})$ and $(\mathrm{M}+\mathrm{P})$ significantly reduced paw diameter compared to the control group, although neither group was found to be superior (Figure 1). The respective $p$-values are shown on Table 1. Dunn's posttest was applied after the above analysis, and the same inferences remained.

MTT colorimetric assay: The absorbance values obtained from the first row of wells (line A) of the plates (corresponding to the undiluted Leishmania concentration from the paws) were used for comparing the groups, using the Wilcoxon test. Our results (Figure 2, Table 1) indicate that there was a significantly lower concentration of Leishmania in the ( $\mathrm{M}+\mathrm{P})$ group, compared to the (M) group.

Analysis of cultures: Cultures obtained on D13 were positive in all 6 samples from group (C), indicating the presence of infection. In group (M), 1 of 6 cultures was positive, and in group $(\mathrm{M}+\mathrm{P}), 2$ of 6 were positive. Fisher's t-test only detected a significant difference between group (C) and (M) (Table 1).

Analysis of slides: Parasites could only be detected visually in the control group (C). All slides from the other groups showed no signs of parasites.

We chose not to use N-methyl glucamine (the first line of treatment for Leishmania infection), since we know that miltefosine has the same efficacy in Leishmania amazonensis ${ }^{4}$, and our goal was to test whether a combination of oral drugs would provide a better response to treatment. When we analyzed the paw diameters, we adopted a significance (alpha) level of $5 \%$, and both treatment groups $(\mathrm{M}, \mathrm{M}+\mathrm{P})$ were significantly smaller $(\mathrm{p}<0.05)$ than group $(\mathrm{C})$ on D6 and D12. However, there was no significant difference between the two treatment groups, and thus there is no evidence that either is superior. These results may indicate that a longer period is required paw

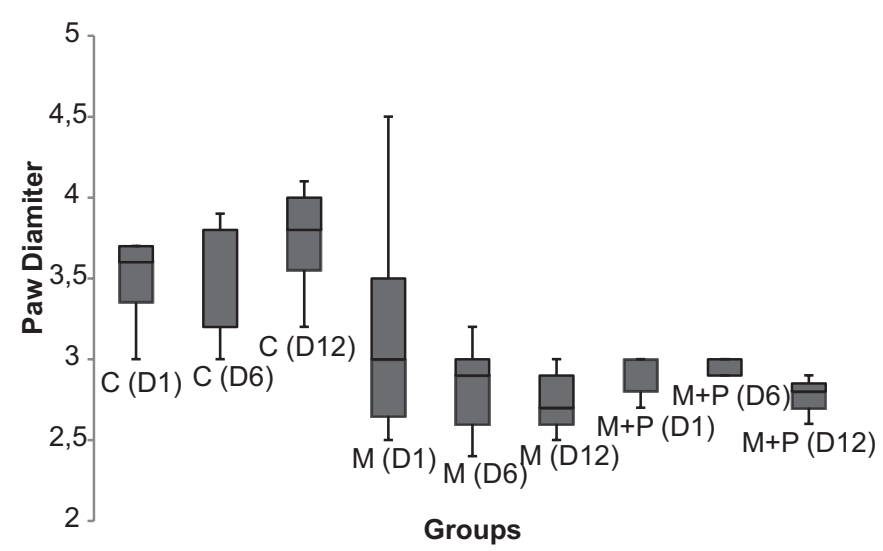

FIGURE 1 - Paw diameter over time. Paw diameters were measured at three time points (D1, D6, and D12). D1 represents diameters before initiating treatment, while D6 and D12 represent diameters on the sixth and on the twelve day of treatment. M: miltefosine; M+P: miltefosine plus pentoxifylline; $\mathbf{C}$ : control. 
TABLE 1 - Analysis of experimental parameters.

\begin{tabular}{|c|c|c|c|c|c|c|c|}
\hline Parameter of analysis & Group C & Group M & Group $\mathrm{M}+\mathrm{P}$ & Time & Groups & Average number* & $p$-value \\
\hline & & & & & $\mathrm{C}$ vs $\mathrm{M}+\mathrm{P}$ & 7.33 & $<0.01$ \\
\hline & & & & & $M$ vs $M+P$ & & 0.92 \\
\hline & $3.742 \pm 0.315$ & $2.766 \pm 0.176$ & $2.733 \pm 0.124$ & D12 & $\mathrm{C}$ vs $\mathrm{M}$ & & $<0.01$ \\
\hline & & & & & $\mathrm{M}$ vs $\mathrm{M}+\mathrm{P}$ & & 0.65 \\
\hline \multirow[t]{3}{*}{ Absorbance of A line } & $1.074 \pm 0.198$ & $0.406 \pm 0.103$ & $0.221 \pm 0.110$ & D13 & $\mathrm{C}$ vs $\mathrm{M}$ & & $<0.01$ \\
\hline & & & & & $\mathrm{C}$ vs $\mathrm{M}+\mathrm{P}$ & 7.33 & $<0.01$ \\
\hline & & & & & $\mathrm{M}$ vs $\mathrm{M}+\mathrm{P}$ & & $<0.01$ \\
\hline \multirow[t]{3}{*}{ Positive cultures (n) } & 6 & 1 & 2 & D13 & $\mathrm{C}$ vs $\mathrm{M}$ & & 0.01 \\
\hline & & & & & $\mathrm{C}$ vs $\mathrm{M}+\mathrm{P}$ & 7.33 & 0.06 \\
\hline & & & & & $\mathrm{M}$ vs $\mathrm{M}+\mathrm{P}$ & & 0.99 \\
\hline
\end{tabular}

C: control group; $\mathbf{M}$ : miltefosine group; $\mathbf{M}+\mathbf{P}$ : miltefosine plus pentoxifylline group; $\mathbf{D}:$ days after initiating treatment; vs: versus. *Average number of animals in the three groups.

diameter to indicate healing and regression of local edema and infiltration, even in the absence of viable parasites. Secondary infections and local trauma caused by the mice's infighting also cannot be discarded.

The MTT colorimetric test indirectly assesses cellular mitochondrial activity and cellular viability, and is relatively immune to observer bias. The absorbance value increases with higher concentrations of formazan (a product of mitochondrial MTT conversion) ${ }^{14}$, and since there is no cutoff point, a direct
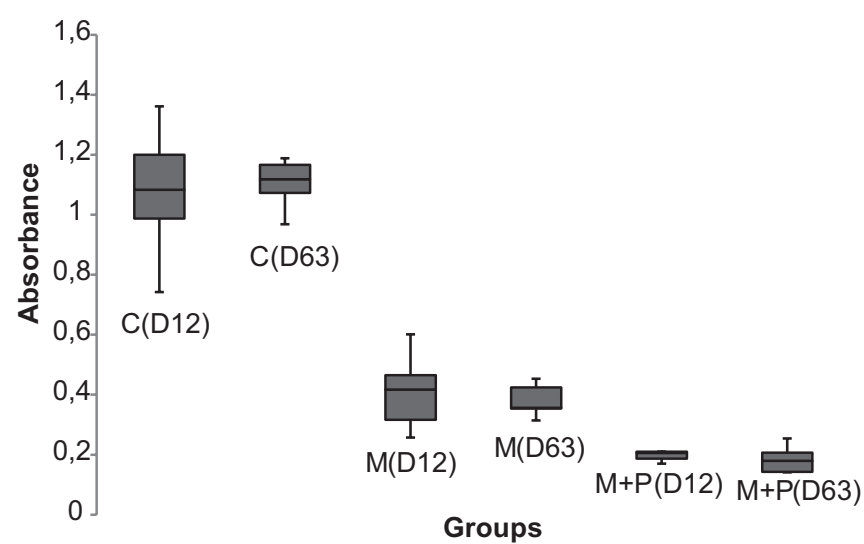

FIGURE 2 - Methylthiazol tetrazolium absorbance. D: days after initiating treatment; $\mathbf{C}$ : control; $\mathbf{M}$ : miltefosine; $\mathbf{M + P}$ : miltefosine plus pentoxifylline. comparison between the effects of the two treatments can be made. Although our primary goal was to determine which of the treatments eliminated the largest number of parasites, we chose not to monitor the mice until the absolute disappearance of parasites and lesions, since the resulting experiment would be burdensomely long. We found that combined treatment $(\mathrm{M}+\mathrm{P})$ was more effective that $(\mathrm{M})$ alone, when both were compared to group $(\mathrm{C})$, as evidenced by the lower absorbance values. Therefore, the combination of miltefosine and pentoxifylline provided greater leishmanicidal effect than miltefosine monotherapy. The greater effectiveness of the combination treatment was also observed D63, given the persistent leishmanicidal effect, although the sample size was suboptimal (Table 1).

The visual observation of the slides did not concretely contribute to the conclusions of this experiment, as visualization of the amastigotes on the slides was inaccurate, possible caused by low sensitivity or observer error. However, when the cultures were analyzed, we only observed a significant difference $(\mathrm{p}<0.05)$ between $(C)$ and $(M)$. Such an inference contradicts the results our absorbance data, which is more accurate than direct observation. Therefore, various factors, such as contamination bias, sensitivity of cultures (30-50\%), and the number of repetitions of each culture, should have been more adequately addressed, since they may have contributed to inadequate observation measurements. The results also could have been validated by histopathological evaluation, although this technique is less sensitive than other imaging techniques, since the cellular infiltrates can persist for years after treatment as previously described. 
The use of drugs with immunomodulatory effects to treat CL is primarily motivated by the need for alternatives to the standard treatment (pentavalent antimony), and has shown promising results in relapse cases $^{8,15}$. The use of combined pentoxifyllinemiltefosine treatments could help reduced drug resistance to miltefosine, as well as possibly increase the efficacy of treatment ${ }^{8}$. Pentoxifylline has also been shown to have a nephroprotective effect when combined with pentavalent antimonials ${ }^{10}$.

A treatment scheme, preferably using oral administration, is necessary, especially in Brazil, where CL is common in the North and Northeast regions. Treatment is especially critical in the Northeast region, which is plagued by drought, extreme poverty, and limited access to healthcare. Considering the high rates of CL resistance to treatment, our results provide hope that combining drugs with antileishmanial and immunomodulatory effects can be effective in combating microbial resistance, as observed in the multidrug regimens used to treat leprosy and tuberculosis.

Our experiments demonstrate the superiority combined miltefosine and pentoxifylline in the colorimetric assay, which is the most accurate test for evaluating treatment efficacy. Therefore, we conclude that this combination may be more effective than the use of miltefosine alone, in the treatment of $\mathrm{CL}$ due to Leishmania amazonensis in $\mathrm{C} 57 \mathrm{Bl} / 6$ mice. From a statistical point of view, there are no factors that would invalidate the results. However, we did not evaluate the power of the tests, since we were required to terminate the experiments due to ethical considerations. Therefore, it is necessary to perform more tests, using other Leishmania species prevalent in Brazil, such as Leishmania (Viannia) braziliensis and Leishmania (Viannia) guyanensis, to confirm and expand these results.

\section{ACKNOWLEDGMENTS}

We thank Tércio Pereira Rodrigues and Viviane Medeiros, the technicians of the Laboratory of Dermatomycology at Universidade de Brasilia, and the dermatologist Ciro Martins Gomes.

\section{CONFLICT OF INTEREST}

The authors declare that there is no conflict of interest.

\section{FINANCIAL SUPPORT}

This work was supported by National Science and Technology Development Council (CNPq - Conselho Nacional de Desenvolvimento Cientifico e Tecnológico), Brazil. Project \# 484662/2012-0.

\section{REFERENCES}

1. Basu MK, Ray M. Macrophage and Leishmania: an unacceptable coexistence. Crit Rev Microbiol 2005; 31:145-154.

2. Machado PR, Lessa H, Lessa M, Guimarães LH, Bang H, Ho JL, et al. Oral pentoxifylline combined with pentavalent antimony: a randomized trial for mucosal leishmaniasis. Clin Infec Dis 2007; 44:788-793.

3. Lima EB, Porto C, Carneiro da Mota JO, Sampaio RNR, Tratamento da leishmaniose tegumentar Americana. An Bras Dermatol 2007; 82:111-124.

4. Costa-Filho AV, Lucas IC, Sampaio RNR. Estudo comparativo entre miltefosina oral e antimoniato de N-metil glucamina parenteral no tratamento da leishmaniose experimental causada por Leishmania (Leishmania) amazonensis. Rev Soc Bras Med Trop 2008; 41:424-427.

5. Obando D, Widmer F, Wright LC, Sorrell TC, Jolliffe KA. Synthesis, antifungal and antimicrobial activity of alkylphospholipids. Bioorg Med Chem 2007; 15:5158-5165.

6. Paris C, Loiseau PM, Bories C, Bréard J. Miltefosine induces apoptosislike death in Leishmania donovani promastigotes. Antimicrob Agents Chemother 2004; 48:852-859.

7. Wadhone P, Maiti M, Agarwal R, Karnat V, Martin S, Saha B. Miltefosine promotes IFN- $\gamma$-dominated anti-leishmanial immune response. J Immunol 2009; 182:7146-7154.

8. Ponte CB, Alves EA, Sampaio RN, Urdapilleta AA, Kückelhaus CS, Muniz-Junqueira MI, et al. Miltefosine enhances phagocytosis but decreases nitric oxide production by peritoneal macrophages of C57BL/6 mice. Int Immunopharmacol. 2012; 13:114-119.

9. Ribeiro de Jesus A, Luna T, Pacheco de Almeida R, Machado PR, Carvalho EM. Pentoxifylline down modulate in vitro $\mathrm{T}$ cell responses and attenuate pathology in Leishmania and HTLV-I infections. Int Immunopharmacol. 2008; 8:1344-1353.

10. Moura FJ, Leal PP, Souza Furtado R, Muniz-Junqueira MI, Veiga JP. Pentoxifylline prevents the meglumine antimonate-induced renal toxicity in rats, but not that induced by the inorganic antimony pentachloride. Toxicology 2008; 243:66-74.

11. Ministério da Saúde. Brasil. Secretaria de Políticas Públicas de Saúde. Manual de Vigilância da Leishmaniose Tegumentar Americana. $2^{\mathrm{a}}$ edição. Brasília (DF); 2007.

12. Titus RG, Marchand M, Boon T, Louis JA. A limiting dilution assay for quantifying Leishmania major in tissues of infected mice. Parasite Immunol 1985; 7:545-555.

13. Lima HC, Bleyenberg JA, Titus RG. A simple method for quantifying Leishmania in tissues of contaminated mices. Parasitol Today 1997; 13:80-82.

14. Denizot F, Lang R. Rapid colorimetric assay for cell growth and survival. Modifications to the tetrazolium dye procedure giving improved sensitivity and reliability. J Immunol Methods 1986; 89: 271-277.

15. Almeida RP, Brito J, Machado PL, Jesus AR, Schriefer A, Guimarães $\mathrm{LH}$, et al. Successful treatment of refractory cutaneous leishmaniasis with GM-CSF and antimonials. Am J Trop Med Hyg 2005; 73:79-81. 